\title{
Manche mögen's heiß - Der Insektengeruchssinn als Inspiration für biomimetische Brandsensorik
}

\author{
Stefan Schütz ${ }^{1}$, Olaf Kiesewetter ${ }^{2}$, Dieter Kohl ${ }^{3}$ \\ ${ }^{1}$ Forstzoologie \& Waldschutz, Georg-August-Universität Göttingen, Büsgenweg 3, 37077 Göttingen, \\ Deutschland, Stefan.schuetz@forst.uni-goettingen.de. \\ 2 UST Umweltsensortechnik GmbH, Dieselstraße 2, 98716 Geschwenda, Deutschland \\ ${ }^{3}$ Institut für Angewandte Physik, Justus-Liebig Universität Gießen, Heinrich-Buff-Ring 16, 35392 \\ Gießen, Deutschland
}

\begin{abstract}
Zusammenfassung:
Die meisten Insekten ergreifen, wie alle anderen Tiere auch, die Flucht, wenn sie in ihrer Umgebung ein größeres Feuer bemerken. Eine kleine Gruppe von Insekten ist jedoch „pyrophil“ und „liebt“ das Feuer. Diese Käfer fliegen Waldbrände an und unterscheiden dabei zwischen verbranntem und nur erhitztem, nahrhaften Holz für ihre Larven. Dazu nutzt der Käfer insbesondere seinen angepassten Geruchssinn, der inn durch die Wahrnehmung von spezifischen Markersubstanzen über Ort und Art des Brandes informiert. Vier solcher Markersubstanzen, die sowohl vom Käfer empfindlich gerochen als auch reproduzierbar bei unterschiedlichen Temperaturen von erhitztem Kiefernholz freigesetzt werden, konnten mittels gaschromatographischer Messungen mit paralleler Detektion durch ein Massenspektrometer und einen Elektroantennographen identifiziert werden. Am Beispiel der Geruchswahrnehmung des Schwarzen Kiefernprachtkäfers Melanophila acuminata wurden so biomimetische Brandsensoren zur sensorgestützten Optimierung der Prozesskontrolle in einem industriellen Holzpartikeltrockner entwickelt. Eine Erhöhung der Trocknerluft-Ausgangstemperatur von $150^{\circ} \mathrm{C}$ auf $155^{\circ} \mathrm{C}$ erbringt bei einer Erhöhung des Energieeinsatzes von 3\% eine Steigerung der Trockengeschwindigkeit von 12\%. Da die Gefahr einer Selbstentzündung in diesem Temperaturbereich steigt, ist für eine solche Erhöhung der Energieeffizienz eine verbesserte Branderkennung nötig. Für die notwendige empfindliche Detektion der identifizierten Markersubstanzen wurden nanoporöse Wolframoxid- Halbleiter-Gassensoren entwickelt, die besonders sensitiv und selektiv auf diese Markersubstanzen reagieren.
\end{abstract}

Key words: Waldbrand, feuerliebende Käfer, Holzpartikeltrocknung, Holzfaserplatten, nanoporöses Wolframtrioxid, biomimetische Halbleiter-Gassensoren.

\section{Das Biologische System}

Wälder sind komplexe, langlebige Ökosysteme, die unterschiedliche Entwicklungszustände von der Entstehung bis zum Zerfall durchlaufen. Die wesentlich kurzlebigeren Insekten spielen bei dieser Entwicklung eine wichtige Rolle und sind oftmals hochspezifisch an bestimmte Entwicklungszustände des Waldes angepasst. In sämtlichen Klimaten ist der Waldbrand ein wichtiger Übergang zwischen spezifischen Entwicklungszuständen des Waldes. Das Vorkommen mancher Pflanzen- und Tierarten hängt sogar vom regelmäßigen Auftreten von Waldbränden ab. „Pyrophile“, das heißt „Feuer liebende", Insekten sind ein Beispiel für eine solche Spezialisierung. Als mobile Organismen mit hoher Vermehrungsrate besiedeln sie unmittelbar nach dem Waldbrand die verbliebene Biomasse und leisten so einen wichtigen Beitrag zur Aufrechterhaltung der Stoffkreisläufe. Da Waldbrände jedoch räumlich und zeitlich unregelmäßig verteilt auftreten, stellt sich die Frage, wie pyrophile Insekten diese aus großer Entfernung auffinden können. Der in der gesamten nördlichen Hemisphäre verbreitete pyrophile Schwarze Kiefernprachtkäfer Melanophila acuminata (Abbildung 1) fiel schon 1913 bei Waldbränden in Kalifornien auf, wo er durch massenhaftes Schwärmen nahe des Feuers Feuerwehrleute beim Löschen behinderte [1]. Käfer beiderlei Geschlechts treffen hart an der Flammenfront zusammen, wo man es als Mensch kaum noch aushält. Häufig kommt es schon zu Paarungen, wenn die Flammen noch lodern. Ist das Feuer verloschen, legen die Weibchen ihre Eier unter die Rinde der verbrannten Bäume. Nach dem Schlüpfen fressen die Larven zunächst in der nahrhaften, durch die Hitze schon „vorgegarten“ Bastschicht, um dann später tief ins Holz 
vorzustoßen. Die Larven können sich ausschließlich auf brandgeschädigten Bäumen ohne funktionsfähige Abwehr entwickeln und brauchen 1-3 Jahre bis zum ausgewachsenen Insekt [2].

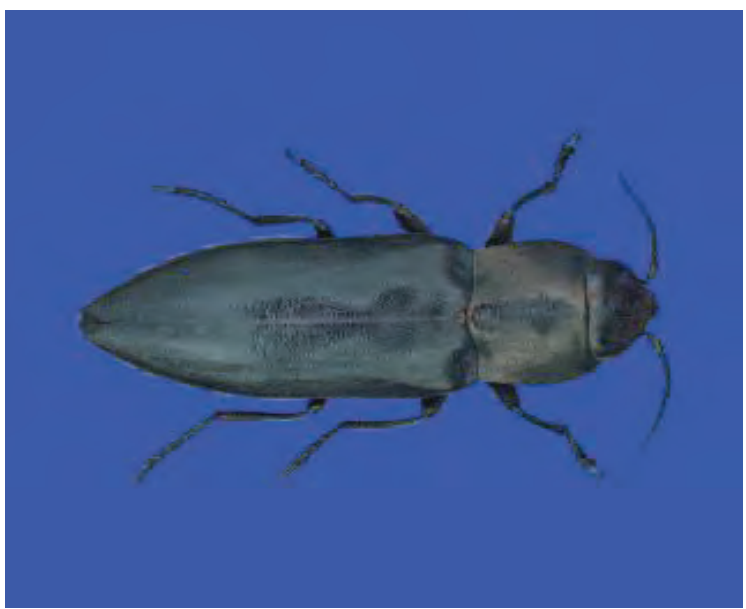

Abb. 1. Der Schwarze Kiefernprachtkäfer (Melanophila acuminata).

Wie gaschromatographische Messungen mit paralleler Detektion durch ein Massenspektrometer und einen Elektroantennographen (GCMS/EAD) [3] zeigen konnten, finden die Käfer Waldbrände mittels ihres Geruchssinns für brandspezifische Duftstoffe in Kombination mit Infrarot-Grubenorganen am Thorax [4, 5]. Die Antennen von M. acuminata können GuaiakolVerbindungen im Rauchgas besonders empfindlich nachweisen (bis $1 \mathrm{ppbv}$ ). Das heißt, dass ein einzelner auf $2 \mathrm{~m}$ Höhe angekohlter Kiefernstamm bei schwachem Wind noch in über $1 \mathrm{~km}$ Entfernung von dem Käfer zu riechen ist. Über eine ganze Palette von Guajakol-Verbindungen ist der Schwarze Kiefernprachtkäfer in der Lage, unterschiedliche Arten von Bränden zu differenzieren, da diese Verbindungen bei unterschiedlichen Brandtemperaturen und Sauerstoffversorgung entstehen. In Feldexperimenten konnte gezeigt werden, dass ein nur wenige Bäume $\left(5 \mathrm{~m}^{3}\right.$ Holz) umfassender Brand noch in über $2 \mathrm{~km}$ Entfernung durch einen Biosensor auf der Basis von $M$. acuminata Antennen detektiert und über den Konzentrationsgradienten mit Hilfe der Windrichtung durch einen dichten Waldbestand zu seinem Ausgangspunkt zurückverfolgt werden konnte.

Nach erfolgreichem Auffinden der Brandfläche und nachfolgender Paarung steht das Weibchen nun vor der Frage, an welchen Baum sie ihre Eier ablegen soll, um ihrem Nachwuchs die besten Chancen zu geben. Oberflächlich verrußte Bäume sind von solchen, die bis zum Kern verkohlt sind, optisch nicht zu unterscheiden. Optimale Bedingungen findet der Nachwuchs jedoch nur in Bäumen, deren Bast und Holz zwar erhitzt und damit vorzersetzt aber noch nicht verkohlt ist. Aufgrund der beginnenden thermischen Aufspaltung von Holzbestandteilen wie Hemizellulosen und Zellulosen setzt Holz in Erhitzungsstadien vor der Entzündung FuranVerbindungen frei, die vom Käfer gerochen werden können [6]. Diese Verbindungen werden zwar nicht so empfindlich aber hochspezifisch wahrgenommen und dienen damit der Orientierung des Käfers auf der Brandfläche.

Die Käfer haben häufig nach langem Anflug des Waldbrandes, Kopulation und Eiablage ihre Energiereserven weitgehend verbraucht. Wenn sie diese Energiereserven nicht auffüllen können, müssen die Käfer auf der Brandfläche sterben. Die außerhalb der Brandsaison räuberisch lebenden Käfer können auf der Brandfläche jedoch kaum Beute finden, da sämtliche nicht pyrophilen Tiere vor dem Waldbrand geflohen sind. Einzige mögliche Nahrungsquelle für die Käfer sind deshalb vom Feuer getötete Tiere, die nicht vollständig verbrannt sind. Auch hier gibt es charakteristische flüchtige Verbindungen, die Käfer zu ihrer Nahrung führen können - der Geruch von gebratenem Fleisch.

\section{Die Inspiration}

Die klassische Art der Detektion von Schwelbränden durch Gassensoren beruhte auf dem Nachweis der anorganischen Spurengase Wasserstoff, Kohlenmonoxid und Stickstoffdioxid [7]. Dies brachte jedoch das Problem der Abgrenzung von Schwelbränden von anderen Verbrennungsprodukten, wie sie durch den Betrieb von Benzin- oder Dieselmotoren freigesetzt werden, mit sich. Das heißt, eine Differenzierung nach der Art des brennenden Materials wurde notwendig - (Holz, Kohle, Gras, Papier etc.), außerdem war eine schnelle Differenzierung früher und später Stadien eines Brandes sowie von Schwelbränden, offenem Feuer und verbliebenen Glutnestern erwünscht. Ein Blick auf den Schwarzen Kiefernprachtkäfer, der anhand der Fernwahrnehmung von GuaiakolVerbindungen pyrolytisch zersetztes Lignin von Holz klar von brennendem Gras oder Benzin unterscheiden konnte, lieferte die Inspiration: Die Ergänzung der klassischen Festkörpersensor-Arrays durch einen Sensor, der spezifisch Guaiakol-Derivate detektieren und so Abgase von Verbrennungsmotoren von Schwelgasen aus Lignin-haltigem Material, wie Holz oder Braunkohle zu unterscheiden [8]. 
Bei der Nutzung nachwachsender Rohstoffe für die Fertigung moderner Verbundmaterialien spielt Holz für die Fertigung von Holzfaserplatten eine besondere Rolle. Die energieintensive Trocknung der Holzfasern vor der Verarbeitung ist hier ein wichtiger Parameter für die ökonomische und ökologische Bewertung dieser Produkte. Die Energieeffizienz dieses Verfahrens kann durch eine Erhöhung der Trocknungstemperatur verbessert werden, doch steigt dann auch die Gefahr einer spontanen Selbstentzündung der Holzpartikel. Eine Erhöhung der TrocknerluftAusgangstemperatur von $150^{\circ} \mathrm{C}$ auf $155^{\circ} \mathrm{C}$ erbringt bei einer Erhöhung des Energieeinsatzes von 3\% eine Steigerung der Trockengeschwindigkeit von 12\% (Abbildung 2). Da die Gefahr einer Selbstentzündung in diesem Temperaturbereich steigt, ist für eine solche Erhöhung der Energieeffizienz eine verbesserte Erfassung der strukturellen Stabilität der Holzpartikel nötig.

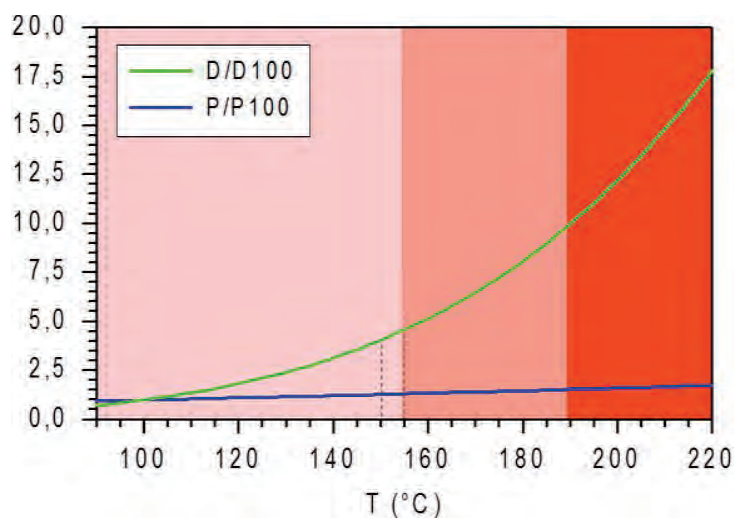

Abb. 2. Vergleich der Temperaturabhängigkeit der einzusetzenden Heizleistung (blau) und der Diffusion von Wasser in Holz (grün) als geschwindigkeitsbestimmender Schritt des Trocknungsprozesses. Die unterschiedlichen Rottöne markieren die Zonen des Sicherheitsbereichs bis zum Flammpunkt von Holzspänen $\left(190^{\circ} \mathrm{C}\right)$ an Luft [10].

Ein Blick auf den Schwarzen Kiefernprachtkäfer, der anhand der Wahrnehmung von Furan-Verbindungen pyrolytisch zersetzte Hemizellulose und Zellulose von Holz klar von entzündetem Holz oder wenig erhitztem Holz unterscheiden konnte, lieferte die Inspiration: Eine sensorgestützte Optimierung der Prozesskontrolle auf der Basis der Detektion von Vorbrandstufen-typischen FuranVerbindungen, wie sie vom Schwarzen Kiefernprachtkäfer für die Auswahl des geeignetsten Eiablageplatzes genutzt werden [9].
Bei der Entwicklung innovativer Küchenprodukte ist die automatische Erkennung des Garzeitpunktes von Fleisch ein noch nicht befriedigend gelöstes Problem. Wie kann der Übergang vom zart gegartem über scharf angebratenem zu verbranntem Fleisch detektiert werden? Ein Blick auf den Schwarzen Kiefernprachtkäfer, der anhand der Wahrnehmung spezifischer Verbindungen garprozessähnlich zersetzte Proteine und Fette von Fleisch klar von verbranntem oder wenig erhitztem Fleisch unterscheiden konnte, liefert die Inspiration: Eine sensorgestützte Optimierung der Garprozessführung im heimischen Herd.

\section{Die technische Sensorik}

Ein bionisches Sensorsystem auf der Basis der von Melanophila acuminata wahrgenommenen Volatilen sollte mit hinreichender Zuverlässigkeit schnell Messdaten liefern, die in der Brandfrühwarnung in Lagern oder im industriellen Verarbeitungsprozess effizient genutzt werden können. Die dann folgende Herausforderung besteht in der technischen Umsetzung.

Die Vorteile von MOS-Halbleitergassensoren gegenüber anderen Gassensorprinzipien sind insbesondere hohe Empfindlichkeiten gegenüber den jeweiligen Zielsubstanzen, Miniaturisierbarkeit, lange Lebensdauer und die Möglichkeit, sowohl in Klein- als auch in Großserienstückzahlen kostengünstig zu produzieren.

Das Funktionsprinzip von Halbleitergassensoren beruht auf der Leitfähigkeitsänderung einer sensitiven Halbleiterschicht bei der Beaufschlagung von Gasen. Sensitive Schichten reagieren auf reduzierende Gase mit der Verringerung des Widerstands und auf oxidierende Gase mit einer Widerstandserhöhung. Der jeweilige Detektionsbereich des Gassensorelementes hängt u.a. vom eingesetzten Sensortyp und der Art des zu messenden Gases ab. Die sensitiven Schichten müssen beheizt werden. Dazu ist in dem Halbleitergassensor ein Heizer integriert. Der Widerstand des Platin-Heizers hat einen definierten Temperaturkoeffizienten, wodurch die Regelung der Arbeitstemperatur des Sensors ermöglicht wird. Somit können Schwankungen der Umgebungstemperatur weitestgehend kompensiert und die Sensitivität des Sensors gezielt beeinflusst werden. Die Selektivität eines Gassensors hinsichtlich der Zielsubtanzen kann gezielt durch die Eigenschaften der jeweiligen gassensitiven Schicht(en), (Schichtmaterialien und deren Struktur, Dotierungen etc.) gesteuert werden. 
Weitere bzw. zusätzliche Möglichkeiten ergeben sich z. B. durch die Steuerung der Betriebsmodi. Durch den gezielten Aufbau von Mehrfachgassensoren (Gassensorarrays) ist dieser Sensortyp für komplexe parallele Detektionsanwendungen für verschiedene Zielsubstanzen geeignet. In Abhängigkeit des Sensordesigns und des eingesetzten Herstellungsverfahrens (Substrat-/Chiptechnologie etc.) sind auch Mehrfachgassensoren vergleichsweise kostengünstig produzierbar. Vorteil dieser Sensortechnologie ist damit insbesondere auch die vergleichsweise einfache und kostengünstige applikationsspezifische Auslegung von Gassensoren [5].

Beim Verbrennen und Verschwelen von Holz oder Braunkohle entstehen unter anderem Methoxy-Phenole [1]. Zinndioxid-basierte Sensorwirkschichten erwiesen sich als besonders geeignet für die Detektion von Methoxy-Phenolen wie Guaiakol- Verbindungen [11]. Diese Wirkschichten wurden mit dem GCMS/SOMSA-Verfahren analysiert [12]. Mittels dieses Verfahrens werden die Halbleitergassensoren, analog zu den Messungen der Depolarisationsreaktionen der Antennen von Melanophila acuminata, auf die flüchtigen Bestandteile des erhitzten und schwelenden Holzes getestet. Die Ergebnisse der GC-MS/SOMSA-Messungen zeigen, auf welche Stoffe aus dem Stoffgemisch des schwelenden Holzes die Sensoren eine Reaktion aufweisen. Zusammen mit den Messungen mit GC-MS/EAD konnten dann die Sensorwirkschichten gezielt auf die entsprechenden Stoffe oder Stoffgruppen aus schwelender Braunkohle angepasst werden.

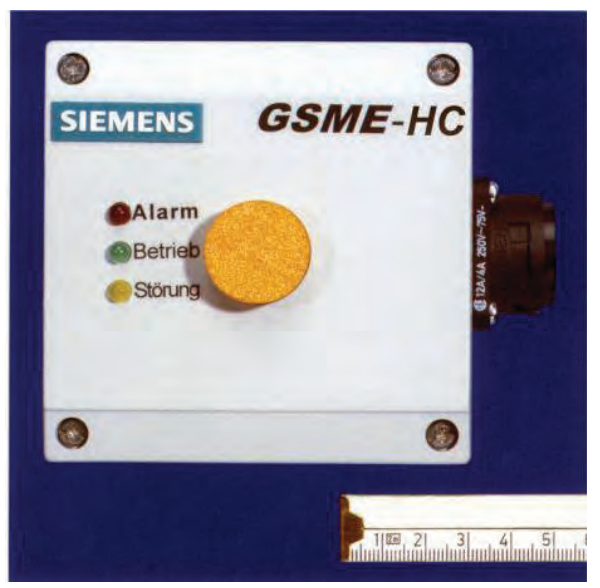

Abb. 3: Schwelbrandsensorsystem für die Brandfrühwarnung in Braunkohlelagern [8]

Der so optimierte Sensor wies eine hohe Sensitivität und Selektivität für phenolische Strukturen, insbesondere Methoxy-Phenole bei geringer Querempfindlichkeit zu Kohlenwasser- stoffen, Aldehyden, Ketonen und Wasser auf. Dieser Sensor konnte nun mit dem bewährten Schwelbrandsensorarray der Firma GTE kombiniert werden und als industriell nutzbarer Schwelbranddetektor in Braunkohle-bunkern der RWE AG die ersten Jahre Praxistest bestehen (Abb. 3).

Zur Entwicklung der sensitiven Schichten für die Vorbrandwarnung in industriellen Holzpartikeltrocknern (Abb. 4) werden neben bekannten Verfahren, wie Variation der Korngrößen, Dotierungen oder Anpassung von Temperaturprogrammen auch neuartige Materialstrukturierungsmethoden durchgeführt. Hierzu gehören die Synthesen von mesoporösem $\mathrm{WO}_{3}-$ Pulver durch Templatingverfahren und die Nanostrukturierung der Materialoberflächen. Somit können z.B. die Oberflächenpolaritäten der Schichten verändert werden.

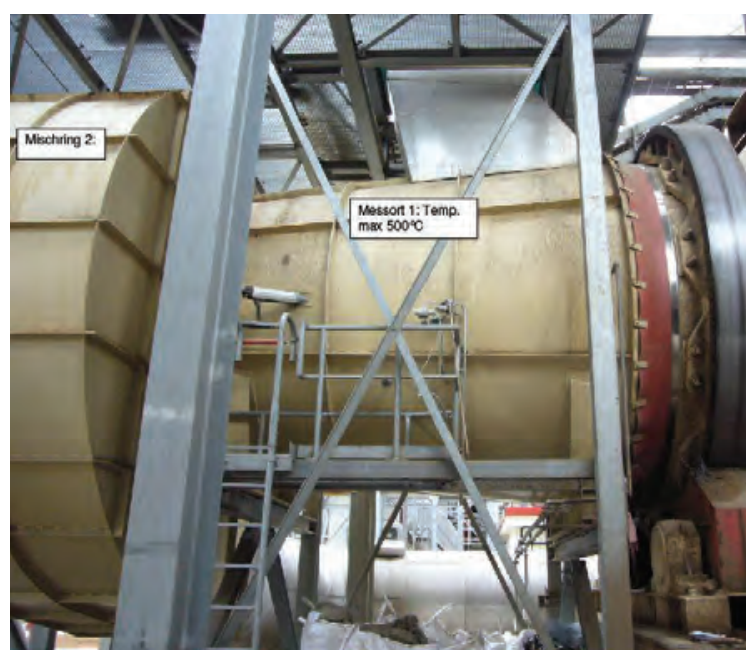

Abb. 4: Trocknereinlaufsbereich/ Trommeltrockner Glunz AG, Werk Nettgau (Foto: Glunz AG)

Aufbauend auf der guten Selektivität der Wolframoxidsensoren gegenüber auftretenden zyklischen Verbindungen [13] erfolgte die Entwicklung mesoporöser $\mathrm{WO}_{3}$-Schichten, die eine gute Sensitivität auf die ermittelten Markervolatile aufweisen und den Anforderungen am Trommeltrockner gerecht werden. Im Template-Casting-Verfahren wurden neuartige mesoporöse $\mathrm{WO}_{3}$-Schichten entwickelt. Dazu wurden poröse SilikaTemplates mit dem Precursor Phosphorwolframsäure gefüllt und unter Temperatureinfluss zu $\mathrm{WO}_{3}$ umgesetzt. Das überflüssige Template wurde anschließend durch Ätzen mit HF entfernt. Diese Syntheseschritte basieren auf den Arbeiten von Rossinyol et al. [14] und resultieren in größeren Teilchen mit einem Durchmesser von $5 \mu \mathrm{m}$, auf denen eine geordnete Substruktur mit Körnern in der Größenordnung von 5-10 nm zu erkennen sind (Abb 5). 
Durch das Driften von Ionen an der Halbleiteroberfläche wird eine Potentialdifferenz aufgebaut, die beim Andocken von reduzierend wirkenden Volatilen verringert wird (Depolarisation). Weil die Gase Grenzflächenzustände bei spezifischen Energien erzeugen, ergibt sich eine Selektivität für bestimmte Gase, wenn die durch das Driften der lonen erzeugte Ausgangspolarisation (Bandverbiegung) entsprechend gewählt ist. Die Polarisation verändert sich beim Halbleitersensor zeitlich und muss deswegen durch Driftzyklen stabilisiert werden [15].

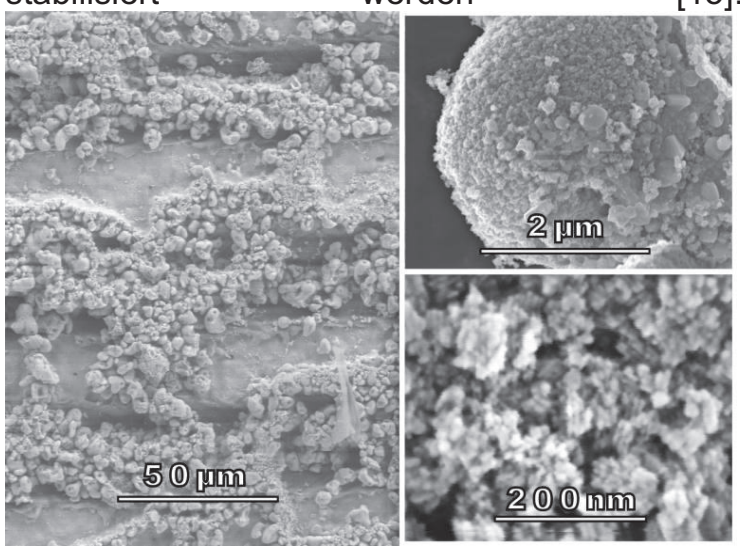

Abb. 5: REM-Aufnahmen des mesoporösen $\mathrm{WO}_{3}$ [9]

Mit diesem Verfahren können simultan verschiedene Sensorwirkschichten untersucht und die Sensorparameter (z.B. Temperatur und Messspannung) optimiert werden. Mit einen Multisignalgewinnungs-verfahren auf der Basis von Temperaturzyklen [7] konnten die Gassensoren die Markervolatile aus dem erhitzten und schwelenden Holz querempfindlichkeitsfrei detektieren und eine Differenzierung der Gasgemische in einer Brandkammer gemäß unterschiedlicher Erhitzungsgrade über die Vorbrandstufe bis zur Selbstentzündung vornehmen (Abb. 6).

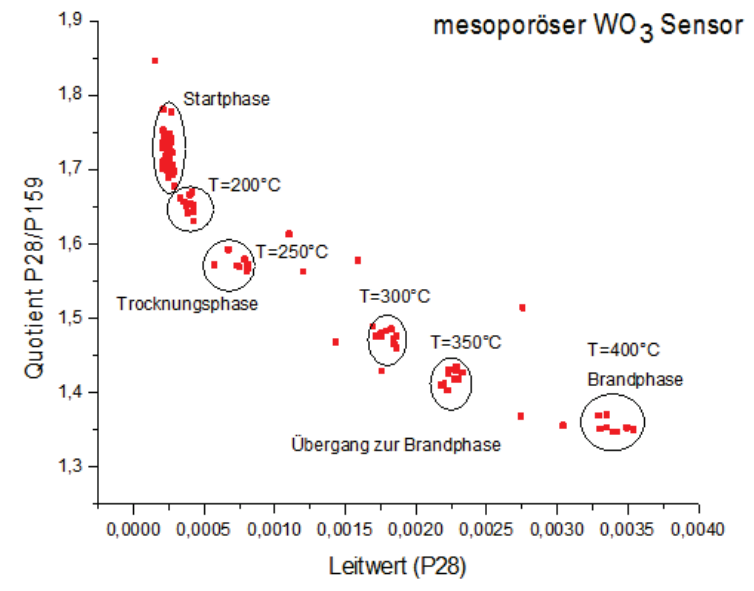

Abb. 6: Trennung der Brandphasen (mesoporöser $\mathrm{WO}_{3}$-Sensor) [10]
Die ersten Sensorprototypen sowie das technische Sensorarray (Abb. 7) zur Charakterisierung und für die Testläufe an unterschiedlichen Positionen des Trommeltrockners der Glunz AG wurden zusammen mit der Firma UST entwickelt und hergestellt.

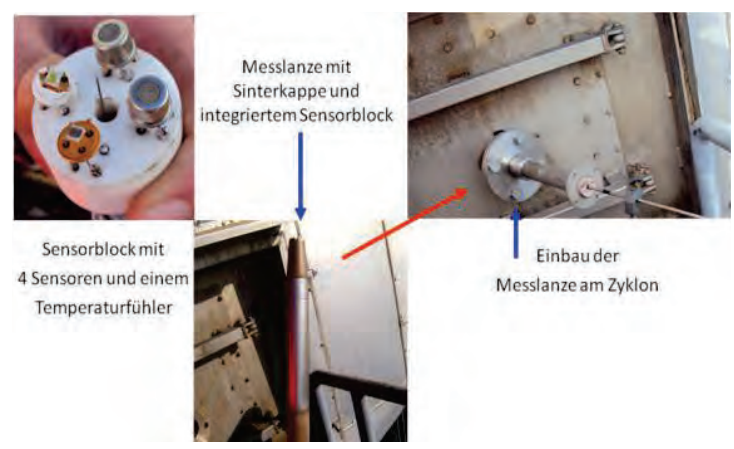

Abbildung 7: Messaufbau am Trommeltrockner [10]

Zur Erfassung von Garprozessen durch Halbleiter- Gassensoren gibt es schon verschiedene Ansätze [12, 16, 17], die mittels Inspiration durch pyrophile Käfer weiter vorangetrieben und auf die Erfassung des Bratens von Fleisch ausgeweitet werden können.

\section{References}

[1] Manee A.H., Observations on Buprestidae at Southern Pines, North Carolina (Coleop.). Entomol. News 24: 167-171 (1913)

[2] Apel K.H.; Zur Verbreitung von Melanophila acuminata DEG. (Col. Buprestidae). Entomol. Nachr. Ber. 33:278-279 (1989)

[3] Weißbecker B., Holighaus G., Schütz S., Gas chromatography with mass spectrometric and electroantennographic detection: -analysis of wood odour by direct coupling of insect olfaction and mass spectrometry, J. Chrom. A 1056:209-216 (2004)

[4] Schütz S., Weißbecker B., Hummel H.E., Apel K.-H., Schmitz H., Bleckmann H., Insect Antennae as a Smoke Detector, Nature, 398:298-299 (1999)

[5] Schmitz H., Schütz S., Waldbrandortung durch Melanophila acuminata, Die spezialisierten Sinnesorgane des "Feuerkäfers", Biologie in unserer Zeit, 30:266-273 (2000)

[6] Paczkowski S., Paczkowska M, Dippel S., Schulze N., Schütz S., Sauerwald T., Weiß A., Bauer M., Gottschald J., Kohl D., The olfaction of a fire beetle leads to new concepts for early fire warning systems. Sensors \& Actuators B-Chemical 183:273$282(2013)$ 
[7] Abschlussbericht zum BMBF-

Verbundprojekt SPAN (Standardised

Primary Aroma Nose) Förderkennzeichen:

16SV1075/0 (2003)

[8] Kohl D. et al., Schieberle P. et al., High

Resolution Gas Chromatographie -

Selective Odorant Measurement by Multi

Sensor Array, Flavour Analysis, Kap. 29,

American Chemical Society, 1998

[9] Paczkowski S., Sauerwald T., Weiß A., Bauer M., Kohl D., Schütz S., "Biomimetic gas sensors for large-scale drying of wood particles", Proceeding of SPIE Volume: 7975: 797505 (2011); doi:

$10.1117 / 12.882421$

[10] Abschlussbericht zum BMBF-

Verbundprojekt BioHot (Bionisch optimierte Holztrocknung) Förderkennzeichen:

01 RB0804 (2012)

[11] Eberheim A., Kohl D., Schieberle P., Tin oxide sensor element for the detection of organic compounds with hydroxyl groups. PCCP 23: 5203-5206 (2003)

[12] Hofmann T., Schieberle P., Krummel C., Freiling A., Bock J., Heinert L., Kohl D., High resolution gas chromatography/ selective odorant measurement by multisensor array (HRGC/SOMSA): a useful approach to standardise multisensor arrays for use in the detection of key food odorants, Sensors and Actuators B, 41:8187 (1997)

[13] Sauerwald T., Skiera D., Kohl C.-D., Selectivity enhancement of gas sensors using non-equilibrium polarisation effects in metal oxide films, Applied Physic A-

Materials Science \& Processing, 87:525529 (2007)

[14] Rossinyol E., Arbiol J., Peiró F., Cornet A., Morante J., Tian B., Bo T., Zhao D.,

Nanostructured metal oxides synthesized by hard template method for gas sensing applications, Sensors and Actuators B: Chemical 109 (1) 57-63 (2005)

[15] Kohl C.D., Electronic noses. In: Waser R (ed.) Nanoelectronics and Information Technology - Advanced Electronic Materials and Novel Devices, Wiley-VCH, Berlin (2003).

[16] Muhl M, Demisch H.U., Becker F., Kohl D., Electronic nose for detecting the deterioration of frying fat - Comparative studies for a new quick test, Eur. J. Lipid Sci. Technol. 102: 581-585 (2000)

[17] Kohl D., Heinert L., Bock J., Hofmann T. Schieberle P., Gas sensors for food aroma during baking and roasting processes based on selective odorant measurements by an array (HRGC/SOMMSA), Thin Solid Films 391: 303-307 (2001) 